\title{
The Survival of Locally Advanced Breast Cancer Patients with Luminal Subtype after Modified Radical Mastectomy
}

\author{
Jefri Adi Kam Sitepu ${ }^{1}$, Marjono Dwi Wibowo ${ }^{2}$, Iskandar Ali $^{3}$ \\ ${ }^{1}$ Department of Surgery, Faculty of Medicine Universitas Airlangga/ Dr. Soetomo General Hospital, Surabaya, \\ Indonesia \\ ${ }^{2}$ Head and Neck Surgery Division, Department of Surgery, Faculty of Medicine Universitas Airlangga/ Dr. \\ Soetomo General Hospital, Surabaya, Indonesia \\ ${ }^{3}$ Oncology Surgery Division, Department of Surgery, Faculty of Medicine Universitas Airlangga/ Dr. Soetomo \\ General Hospital, Surabaya, Indonesia
}

Corresponding Author: Jefri Adi Kam Sitepu

\begin{abstract}
Background: Breast cancer is the leading cause of cancer death in women worldwide. Breast cancer has been classified into several molecular subgroups with different prognostic consequences based on immunohistochemistry and molecular pathology. The prognosis that commonly used is 5-years survival. In this study we aimed to examine the relationship between luminal subtype and 5-year survival rate in patients with early post-mastectomy breast cancer.
\end{abstract}

Methods: We recruited early breast cancer patient who underwent modified radical mastectomy (MRM) in Dr. Soetomo General Hospital (Surabaya, Indonesia) between 2010 2014. Breast cancer tissues were collected during surgery for immunohistochemistry. The patients' 5-years survival data was obtained from medical records and by phone call to the patients or to the close relatives of the patients. Breast cancer subtype was determined based on the result of immunohistochemistry

Results: A total of 84 patients was included in this study. The majority of patients were aged $>40$ years $(72 / 84 ; 85.7 \%)$. There were 39 patients (39/84; 46.4\%) with luminal A subtype and 45 patients (45/84; 53.6\%) with luminal B subtype. Seventy-four patients were diagnosed as invasive ductal carcinoma histologically. Almost all of the patients were able to survive within 5 years $(81 / 84 ; 96.4 \%)$. We found that luminal B had a 1.071 times higher risk of dying within 5 years after therapy than luminal type A, although the analysis did not show significant results $(\mathrm{P}=0.101)$.

Conclusion: Luminal B was the most prevalent breast cancer subtype in Surabaya, Indonesia. The prevalence of breast cancer was higher in patients aged $>40$ years. There was no significant difference between the 5-years survival of luminal A and luminal B subtypes.

Keywords: survival, locally advanced breast cancer, luminal subtype, mastectomy

\section{INTRODUCTION}

Breast cancer is the leading cause of cancer death in women worldwide. Approximately $25 \%$ of all cancer cases in the world are breast cancer, and $15 \%$ of cancer deaths in women are due to breast cancer [1]. GLOBOCAN 2012 estimated that the incidence of female breast cancer in Indonesia was 40.3 per 100,000 and the mortality rate was as high as 16.6 per 100,000 (https://gco.iarc.fr/).

Early breast cancer is defined as stage I and II breast cancer when the tumor size is less than or equal to $5 \mathrm{~cm}$, no regional axillary lymph node metastases, and no distant metastases are found [2]. The expression of the estrogen receptor (ER), progesterone receptor (PR), human epidermal growth factor receptor 2 (HER2/neu), and Ki67 are well-known 
variables that can be used to predict breast cancer prognosis. Breast cancer has been classified into several molecular subgroups with different prognostic consequences based on immunohistochemistry and molecular pathology [3]. Furthermore, intrinsic molecular subtypes, which have been examined primarily in early-stage breast cancer, play a large role in the patterns of failure in breast cancer therapy [4].

Luminal A subtype is the most common breast cancer subtype found and represent up to $60 \%$ of all breast cancer cases [5]. Luminal A have better prognosis than the other subtypes, including Luminal B subtype. The Luminal B sub-type has a higher transcription, proliferation, angiogenesis, and metastatic index compared to the Luminal A subtype. Luminal B is a less commonly found subtype than luminal A, but with higher recurrence rare and lower survival rates compared to luminal A [6].

Breast cancer survival rate reflects the percentage of breast cancer survivors who live 5 years or more after diagnosis. Survival rate in breast cancer decreases with increasing stage. However, there are limited data on the 5-years survival rates in Indonesia. Therefore, in this study we aimed to examine the relationship between luminal subtype and 5-year survival rate in patients with early post-mastectomy breast cancer.

\section{MATERIALS \& METHODS Study participants}

The study was designed as a retrospective cohort study in order to determine the relationship between luminal subtype and 5-years survival in early breast cancer patient. We recruited early breast cancer patient who underwent modified radical mastectomy (MRM) in Dr. Soetomo General Hospital (Surabaya, Indonesia) between 2010-2014. Patient's demographic data was obtained from the medical records. Breast cancer tissues were collected during surgery (modified radical mastectomy/ MRM) for further immunohistochemistry examination. The patient's 5-years survival data was obtained from medical records. The 5-years survival data of patient that was not available in the medical record was collected by phone call to the patients or to the close relatives of the patients in which the contact number was recorded in the medical record.

Ethical approval was obtained from the Ethics Committee of Dr. Soetomo General Hospital (Surabaya, Indonesia) following the guidelines of the Declaration of Helsinki.

\section{Immunohistochemistry and breast cancer subtypes}

Immunohistochemistry examination was performed to the breast cancer tissue that were collected during MRM. Immunohistochemistry was done to examine the expression of estrogen receptor (ER), progesterone receptor (PR), HER2 receptor, and Ki67 receptor. Breast cancer subtype was determined based on the result of immunohistochemistry. Luminal A was defined as patients with ER-positive, increased PR, HER2-negative, and decreased Ki67. Luminal B was defined in patients with immunohistochemistry result: (1) ER-positive, decreased PR or increased Ki67, and HER2-negative or (2) ERpositive, any PR result, HER2-positive, and any Ki67 result. HER2 overexpression was determined when the immunohistochemistry was ER-negative, PR-negative, and HER2positive. Triple negative breast cancer (TNBC) was determined when all ER, PR, and HER2 showed negative result. In this study, we excluded patients with HER2 overexpression and TNBC patients. Patients with luminal A and luminal B were included in this study for further analysis.

\section{Statistical Analysis}

The statistical analysis was performed using the SPSS statistical software package version 23.0 (IBM Corp., Armonk, NY, USA). Discrete variables were tested using the Chi-square test. 
Jefri Adi Kam Sitepu et.al. The survival of locally advanced breast cancer patients with luminal subtype after modified radical mastectomy.

Statistical significance was determined when the P value was less than 0.05 .

\section{RESULT}

\section{Subject's characteristics}

We analyzed the distribution of breast cancer patients who underwent modified radical mastectomy (MRM) between 2010-2014. In a span of 5 years from 2010 to 2014, there were eighty-four breast cancer patients who met the inclusion and exclusion criteria with mean age of 47.5 \pm 6.7 years (range of $31-59$ years). The distribution of patient data in each year is shown in Table 1.

The youngest age of subjects in this study was 31 years and the oldest age was 59 years. Based on the age group, the majority of patients were aged $>40$ years (72/84; 85.7\%) and followed by patients aged $\leq 40$ years $(12 / 84 ; 14.3 \%)$. Our data showed that the prevalence of patients with breast cancer were increasing along with the increasing age.
Based on the immunohistochemistry results, there were 39 patients (39/84; $46.4 \%$ ) with luminal A subtype and 45 patients (45/84; 53.6\%) with luminal B subtype. Based on histopathology examination, breast cancer patients in this study were divided into 3 types: 74 patients with invasive ductal carcinoma (IDC), 6 patients with invasive lobular carcinoma (ILC), and 4 patients with mixed-type. In addition, during the period between 2010 and 2014, there has always been an increase in the number of patients with IDC, from 10 patients (13.5\%) in 2010 to 18 patients $(24.3 \%)$ in 2014 . In terms of outcome, the majority of patients able to survive within 5 years $(81 / 84 ; 96.4 \%)$, and only 3 people died $(3 / 84 ; 3.6 \%)$. From the outcome data search, it was found that the cause of death of the three people was a cause related to the history of breast cancer and not caused by other causes classified as non-medical. In addition, none of the subjects in this study were diagnosed with residual tumors.

Table 1. Breast cancer subjects' characteristics between 2010-2014

\begin{tabular}{|c|c|c|c|c|c|c|}
\hline \multirow[t]{2}{*}{ Characteristic } & \multirow[t]{2}{*}{$\mathbf{n}$} & \multicolumn{5}{|l|}{ Year } \\
\hline & & 2010 & 2011 & 2012 & 2013 & 2014 \\
\hline \multicolumn{7}{|l|}{ Age } \\
\hline$\leq 40$ years & 12 & $2(16.7)$ & $4(33.3)$ & $1(8.3)$ & $1(8.3)$ & 4 (33.3) \\
\hline$>40$ years & 72 & $9(12.5)$ & $10(13.9)$ & $16(22.2)$ & $19(26.4)$ & $18(25.0)$ \\
\hline \multicolumn{7}{|l|}{ Subtype } \\
\hline Luminal A & 39 & $5(12.8)$ & $9(23.1)$ & $6(15.4)$ & 7 (17.9) & $12(56.4)$ \\
\hline Luminal B & 45 & $6(13.3)$ & $5(11.1)$ & $11(24.4)$ & $13(28.9)$ & $10(22.2)$ \\
\hline \multicolumn{7}{|l|}{ Histopathology } \\
\hline IDC & 74 & $10(13.5)$ & $13(17.6)$ & $16(21.6)$ & $17(23.0)$ & $18(24.3)$ \\
\hline ILC & 6 & $1(16.7)$ & $0(0.0)$ & $1(16.7)$ & $1(16.7)$ & $3(50.0)$ \\
\hline Mixed & 4 & $0(0.0)$ & $1(25.0)$ & $0(0.0)$ & $2(50.0)$ & $1(25.0)$ \\
\hline \multicolumn{7}{|l|}{ 5-years survival } \\
\hline Survive & 81 & $11(13.6)$ & 12 (14.8) & $17(21.0)$ & $19(23.5)$ & $22(27.2)$ \\
\hline Not survive & 3 & $0(0.0)$ & $2(66.7)$ & $0(0.0)$ & $1(33.3)$ & $0(0.0)$ \\
\hline
\end{tabular}

\section{Association between patient}

characteristics and 5-years survival rates

We analyzed the association between patient's characteristics with 5-year survival (5-year survival). The data are presented in Table 2. From the aspect of age, it was found that in the age group of $\leq 40$ years, there were 2 patients who did not survive within 5 years and 1 patient in the age group of $>40$ years.
We found that all patients with luminal A type survived 5 years after surgery (39/39; 100\%). There were only 3 luminal B patients who did not survive until 5 years after receiving surgical therapy (3/45; 6.7\%). In terms of histopathological results, 2 patients who died were classified as IDC and 1 patient was classified as ILC. 
Jefri Adi Kam Sitepu et.al. The survival of locally advanced breast cancer patients with luminal subtype after modified radical mastectomy.

Table 2. Association between patient characteristics and 5years survival

\begin{tabular}{|l|l|l|l|}
\hline Characteristics & \multirow{2}{*}{ Total n } & Outcome \\
\cline { 3 - 4 } & & Survive & Not survive \\
\hline Age & & & \\
\hline$\leq 40$ tahun & 12 & $10(83.3)$ & $2(16.7)$ \\
\hline$>40$ tahun & 72 & $71(98.6)$ & $1(1.2)$ \\
\hline & & & \\
\hline Subtype & & & \\
\hline Luminal A & 39 & $39(100)$ & $0(0,0)$ \\
\hline Luminal B & 45 & $42(93.3)$ & $3(6.7)$ \\
\hline & & & \\
\hline Histopathology & & & \\
\hline IDC & 74 & $72(97.3)$ & $2(2.7)$ \\
\hline ILC & 6 & $5(83.3)$ & $1(6.7)$ \\
\hline Mixed & 4 & $4(100)$ & $0(0.0)$ \\
\hline
\end{tabular}

\section{Immunohistochemistry results}

Immunohistochemical examination was performed on tissue pieces obtained at the time of surgery. The distribution of immunohistochemical results from 2010 to 2014 is shown in Table 3. Examination with antibodies to estrogen, progesterone, and Her2neu receptors was then performed. It was found that almost all samples were estrogen receptor (ER) positive (82/84; $97.6 \%)$. The data obtained in this study also showed that 62 patients (62/84; 73.8\%) were progesterone receptor (PR) positive. From the aspect of Her2 receptors, 47 patients were categorized as negative (47/84; 56.0\%) and 37 patients (37/84; $44.0 \%$ ) were categorized as positive, which were divided into: 5 Her2 patients $(+1), 2$ patients Her2 (+2), and 28 Her2 (+3) patients.

Table 3. Immunohistochemistry results

\begin{tabular}{|c|c|c|c|c|c|c|}
\hline \multirow[t]{2}{*}{ Characteristic } & \multirow{2}{*}{ Total n } & \multicolumn{5}{|l|}{ Year } \\
\hline & & 2010 & 2011 & 2012 & 2013 & 2014 \\
\hline \multicolumn{7}{|l|}{ ER } \\
\hline Negative & 2 & $0(0.0)$ & $0(0.0)$ & $1(50.0)$ & $1(50.0)$ & $0(0.0)$ \\
\hline Positive & 82 & 11 (13.4) & 14 (17.1) & 16 (19.5) & 19 (23.2) & $22(26.8)$ \\
\hline \multicolumn{7}{|l|}{ PR } \\
\hline Negative & 22 & $3(13.6)$ & $3(13.6)$ & $5(22.7)$ & $7(31.8)$ & $4(18.3)$ \\
\hline Positive & 62 & $8(12.9)$ & $11(17.7)$ & $12(19.4)$ & $13(21.0)$ & $18(29.0)$ \\
\hline \multicolumn{7}{|l|}{ Her2 } \\
\hline Negative & 47 & $6(12.8)$ & 12 (25.5) & 9 (19.1) & $10(21.3)$ & $10(21.3)$ \\
\hline $1+$ & 5 & $1(20.0)$ & $0(0.0)$ & $0(0.0)$ & $0(0.0)$ & $4(80.0)$ \\
\hline $2+$ & 4 & $1(25.0)$ & $0(0.0)$ & $2(50.0)$ & $1(25.0)$ & $0(0.0)$ \\
\hline $3+$ & 28 & $3(10.7)$ & $2(7.1)$ & $6(21.4)$ & $9(32.1)$ & $8(28.6)$ \\
\hline
\end{tabular}

Association between breast cancer subtypes and 5-years survival rates

We also analyzed the association between the luminal subtype of breast cancer and the risk of death within 5 years after surgical therapy (Table 4). Using regression analysis, it was found that luminal B had a 1.071 times higher risk of dying within 5 years after therapy than luminal type $\mathrm{A}$, although the analysis did not show significant results $(\mathrm{P}=0.101,95 \%$ $\mathrm{CI}=0.991-1.158)$.

Table 4. Association between breast cancer subtypes and 5-years survival rates

\begin{tabular}{|l|l|l|l|l|l|l|}
\hline Luminal subtype & $\mathbf{n}$ & \multicolumn{2}{|c|}{ 5-years survival } & OR & $\mathbf{9 5 \%}$ CI & P Value \\
\cline { 3 - 5 } & & Survive & Not survive & & & \\
\hline Luminal A & 39 & $39(100)$ & $0(0.0)$ & 1 & & \\
\hline Luminal B & 45 & $42(93.3)$ & $3(6.7)$ & 1.071 & $0.991-1.158$ & 0.101 \\
\hline
\end{tabular}

\section{DISCUSSION}

In this study, it was found that the incidence of breast cancer was more common in the age group of more than 40 years compared to the age group of less than 40 years. This shows that the incidence of breast cancer is associated with older age. This result was consistent with previous studies reported that breast cancer was associated with age, and its prevalence increases along with the increasing age [7]. There are various factors that cause the high incidence of cancer in older age, including changes in hormonal levels in older women and a lower immune system. However, although the incidence of breast cancer 
under the age of 40 is smaller than in patients over the age of 40 , patients who suffer from breast malignancy at a younger age tend to have cancer with a more aggressive nature Therefore, early detection of breast cancer at a young age is very important to improve overall survival. The incidence of breast cancer at a young age is now also reported to be increasing. The awareness to carry out early screening for breast cancer may increase the number of cases detected. However, at a young age, the sensitivity of mammography as the gold standard for breast cancer screening tends to decrease, therefore young women at high risk should undergo regular mammograms with the addition of magnetic resonance imaging (MRI) examinations [8] to increase the accuracy of detection. Although it has disadvantages such as high cost and low specificity, MRI can be used as an option for breast cancer screening modality because it has a very high sensitivity rate, especially when combined with mammography [9].

Epidemiologically, breast cancer subtypes with positive hormonal status are the type with the highest number of cases. From previous study in the Unites States of America, the most common breast cancer found in the USA was luminal A subtype (72.6\%), followed by triple-negative breast cancer (11.3\%), luminal B subtype (11.2\%), and HER2 overexpression type 4.8\% [10]. However, from this study it was found that the most common subtype found was Luminal B. Our result was in concordance with previous research conducted in Indonesia which stated that luminal B subtype was the most prevalent subtype in Indonesia (43.2\%), followed by HER2overexpression subtype (17\%), TNBC (18.1\%), and luminal A subtype (21.7\%) [11]. The differences in the distribution of breast cancer subtypes in various studies from different regions in the world showed that the distribution of breast cancer subtypes is strongly influenced by geographical factor. This shows the importance of epidemiological studies that describe the distribution of breast cancer subtypes because these data can help the clinicians in daily practice, especially in situations where immunohistochemistry examination cannot be performed. The clinicians can have an idea of the best treatment options that can be given based on epidemiological data in that population.

In terms of histopathological diagnosis, there were 3 major groups of breast cancer types, namely IDC, ILC, and mixed-type. This study showed that the most common type is the IDC type. The incidence of IDC even far exceeds the incidence of ILC and mixed-types. Previous study was in concordance with our results and showed that IDC was the most common histopathological diagnosis of breast cancer in a population $[12,13]$. In general, IDC is reported as the most common type of invasive breast cancer found worldwide. This is a good thing, because various studies report that IDC is the histopathological type of breast cancer that gives the best clinical response to neoadjuvant chemotherapy (NAC) when compared to ILC [14]

In this study also analyzed the relationship between patient characteristics and the type of breast carcinoma to the outcome of the patient, in this case whether the patient was able to survive until 5 years after receiving mastectomy or died within 5 years after surgery. However, we did not find any significant association statistically between breast cancer subtypes and patient outcomes. This indicates that both luminal A and luminal B subtypes do not have a significant difference in terms of the patient's outcome in 5 years. Most studies stated that luminal B subtype had more slightly worst outcome compared to luminal A subtype. One of them was the study of Cho et al., which stated that luminal B subtype had higher proliferative gene expression and lower survival rates than the luminal A subtype [15]. The non-significant results in this study might be caused by the relatively small number of subjects included in this study. A more comprehensive study with a larger number of patients is needed in 
order to describe the association between breast cancer subtypes and 5-years survival of patients with breast cancer. Similar conclusion was also obtained after analyzing the association between other factors, such as age and histopathological diagnosis on the clinical outcome of patients.

There are several limitations in this study. First, the number of samples in this study is relatively small, therefore the statistical analysis was not able to show results with significant power. Further research using a larger sample size and conducted in a longer research period might be important in the future. Second, in this study the authors included early-stage breast cancer patients, both those who had undergone chemotherapy and those who had not undergone chemotherapy in accordance with the breast cancer treatment protocol from ESMO. This may cause bias in the analysis.

\section{CONCLUSION}

Luminal B was the most prevalent breast cancer subtype in Surabaya, Indonesia. The prevalence of breast cancer was higher in patients aged $>40$ years. There was no significant difference between the 5years survival of luminal A and luminal B subtypes.

\section{Acknowledgement: None}

\section{Conflict of Interest: No competing}

interests declared.

\section{Source of Funding: None}

\section{Ethical Approval: Approved}

\section{REFERENCES}

1. Kim HY, Kim TH, Yoon HK, Lee A (2019) The Role of Neutrophillymphocyte Ratio and Plateletlymphocyte Ratio in Predicting Neoadjuvant Chemotherapy Response in Breast Cancer. J Breast Cancer 22: 425438.
2. Koh J, Kim MJ (2019) Introduction of a New Staging System of Breast Cancer for Radiologists: An Emphasis on the Prognostic Stage. Korean J Radiol 20: 6982.

3. Yersal O, Barutca S (2014) Biological subtypes of breast cancer: Prognostic and therapeutic implications. World J Clin Oncol 5: 412-424.

4. Arvold ND, Taghian AG, Niemierko A, Abi Raad RF, Sreedhara M, et al. (2011) Age, breast cancer subtype approximation, and local recurrence after breastconserving therapy. J Clin Oncol 29: 3885-3891.

5. Al-Thoubaity FK (2020) Molecular classification of breast cancer: A retrospective cohort study. Ann Med Surg (Lond) 49: 44-48.

6. Hashmi AA, Aijaz S, Khan SM, Mahboob $\mathrm{R}$, Irfan $\mathrm{M}$, et al. (2018) Prognostic parameters of luminal A and luminal B intrinsic breast cancer subtypes of Pakistani patients. World J Surg Oncol 16: 1.

7. Lima SM, Kehm RD, Terry MB (2021) Global breast cancer incidence and mortality trends by region, age-groups, and fertility patterns. EClinicalMedicine 38: 100985.

8. McGuire A, Brown JA, Malone C, McLaughlin R, Kerin MJ (2015) Effects of age on the detection and management of breast cancer. Cancers (Basel) 7: 908929.

9. Cho N, Han W, Han B-K, Bae MS, Ko ES, et al. (2017) Breast Cancer Screening With Mammography Plus Ultrasonography or Magnetic Resonance Imaging in Women 50 Years or Younger at Diagnosis and Treated With Breast Conservation Therapy. JAMA Oncology 3: 1495-1502.

10. Acheampong T, Kehm RD, Terry MB, Argov EL, Tehranifar P (2020) Incidence Trends of Breast Cancer Molecular Subtypes by Age and Race/Ethnicity in the US From 2010 to 2016. JAMA Netw Open 3: e2013226.

11. Widiana IK, Irawan H (2020) Clinical and Subtypes of Breast Cancer in Indonesia. 
Jefri Adi Kam Sitepu et.al. The survival of locally advanced breast cancer patients with luminal subtype after modified radical mastectomy.

Asian Pacific Journal of Cancer Care 5: 281-285.

12. Eheman CR, Shaw KM, Ryerson AB, Miller JW, Ajani UA, et al. (2009) The changing incidence of in situ and invasive ductal and lobular breast carcinomas: United States, 1999-2004. Cancer Epidemiol Biomarkers Prev 18: 17631769.

13. Makki J (2015) Diversity of Breast Carcinoma: Histological Subtypes and Clinical Relevance. Clin Med Insights Pathol 8: 23-31.

14. Barroso-Sousa R, Metzger-Filho O (2016) Differences between invasive lobular and invasive ductal carcinoma of the breast: results and therapeutic implications. Ther Adv Med Oncol 8: 261-266.

15. Cho N (2021) Imaging features of breast cancer molecular subtypes: state of the art. J Pathol Transl Med 55: 16-25.

How to cite this article: Sitepu JAK, Wibowo MD, Ali I. The survival of locally advanced breast cancer patients with luminal subtype after modified radical mastectomy. International Journal of Research and Review. 2021; 8(11): 191-197. DOI: https:// doi.org/10.52403/ijrr.20211125 